\title{
Sequential Activation of AMPA Receptors and Glial Cells in a Pain Model of Lumbar Spine Disc Herniation
}

\author{
Dong Gyu Kwak, MD, Dong Gyu Lee, MD, PhD
}

Department of Physical Medicine and Rehabilitation, Yeungnam University College of Medicine, Daegu, Korea

Objective To investigate the glial cell and AMPA (alpha-amino-3-hydroxy-5-methyl-4-isoxazolepropionic acid) receptor activity after surgery for disc herniation pain model.

Methods In total, 83 Sprague-Dawley rats were randomly assigned to the following groups: control ( $\mathrm{n}=16)$, shamoperated $(n=4)$, rats for pain behavior evaluation $(n=3)$, nucleus pulposus-exposed groups for AMPA receptors $(n=30)$, and glial cell $(n=30)$. The rats were tested for mechanical allodynia; immunohistochemical staining for AMPA receptors (GluAl and GluA2) and glial cells (OX-42 and glial fibrillary acid protein [GFAP]) in the spinal dorsal horn was performed on postoperative days 3, 7, and 14.

Results Mechanical withdrawal thresholds decreased after surgery, and this effect was maintained for up to 14 days. Immunohistochemical expression of GluA1 and GluA2 in the spinal dorsal horn had increased quantitatively on postoperative days 3 and $7(\mathrm{p}<0.05)$ to levels similar to that of the controls on postoperative day 14. Moreover, immunohistochemical expression of OX-42 and GFAP showed similar changes to AMPA receptors after surgery. Although the activity of AMPA receptors and glial cells achieved normalcy, the mechanical withdrawal threshold of the hind paw remained decreased 38 days after surgery.

Conclusion The rat model of lumbar disc herniation showed increased expression of AMPA receptor and glial cell activity in the spinal dorsal horn 3 and 7 days after surgery, which deceased to control levels at 14 days. The AMPA receptors and glial cell activations showed similar patterns after disc herniation surgery.

Keywords AMPA receptors, Intervertebral disc displacement, Spinal cord dorsal horn, Inflammation, Synaptic plasticity

Received November 26, 2019; Revised February 10, 2020; Accepted March 11, 2020; Published online September 28, 2020

Corresponding author: Dong Gyu Lee

Department of Rehabilitation Medicine and Spine Center, Yeungnam University College of Medicine, 170 Hyeonchung-ro, Nam-gu, Daegu 42415, Korea. Tel: +82-53-620-3829, Fax: +82-53-620-3270, E-mail: painfree@yu.ac.kr

ORCID: Dong Gyu Kwak (https://orcid.org/0000-0001-8431-7790); Dong Gyu Lee (https://orcid.org/0000-0002-4787-4448)

(c) This is an open-access article distributed under the terms of the Creative Commons Attribution Non-Commercial License (http://creativecommons.org/ licenses/by-nc/4.0) which permits unrestricted noncommercial use, distribution, and reproduction in any medium, provided the original work is properly cited. Copyright $\odot 2020$ by Korean Academy of Rehabilitation Medicine 


\section{INTRODUCTION}

Alpha-amino-3-hydroxy-5-methyl-4-isoxazolepropionic acid (AMPA) receptors participate in the development and maintenance of central sensitization following tissue injury or chronic inflammation [1]. AMPA receptors are ionotropic transmembrane heterotetrameric cation channels consisting of four subunits: GluAl-4. The GluAl and GluA2 subunits are mainly located on the laminae I and II of the spinal dorsal horn, a primary location for nociception and central sensitization $[2,3]$. Peripheral inflammation can upregulate the AMPA receptors within the post-synaptic membranes [4]. The upregulation and trafficking of the GluAl subunit increase excitatory postsynaptic current (EPSC) within the spinal dorsal horn. An increase in EPSC means an increase of nociception. Therefore, investigating the activity of AMPA receptors is crucial for understanding the rapid, activity-dependent interaction of the post-synaptic neurons during peripheral inflammation.

Glial cells interact with the neurons of the dorsal horn via a complex communication network [5]. The activation of nociceptors following the onset of inflammatory pain results in glial response. Activated glial cells release cytokines and chemokines, which trigger the transmission of pain to the post-synaptic neurons in the brain [6]. Astrocytes are in close contact with the neurons, and the positive feedback loop during nociception can cause direct alteration of neuronal communication [6]. Simultaneously, activated glial cells promote the release of anti-inflammatory factors that facilitate the clearance of apoptotic cells and the subsequent halting of pro-inflammatory reactions [7]. Glial cells are regarded as a therapeutic target in the treatment of chronic neuropathic pain. However, temporal changes in glial cell activity and function after disc herniation have not been identified.

Nucleus pulposus (NP) is immunogenic; thus, herniated NP may release a substance capable of inducing an autoimmune response, thereby generating chronic inflammation around the dorsal root ganglia (DRG) [8]. The rat model of lumbar disc pain can be used to induce inflammation around the spinal nerve root and DRG, following the implantation of NP harvested from the tail [9-12]. Inflammation initiates the propagation of action potentials from the peripheral nociceptors and pain perception, which, subsequently, promotes central sensiti- zation of nociception $[4,13]$. Among the biomarkers and cells involved in central sensitization, we investigated the changes in AMPA receptors, as rapidly activated ion channels responding to inflammation and glial cell activation are the key processes involved in maintaining chronic pain.

Previous research reported increased AMPA receptor activity in a pain model of lumbar disc herniation 3 days after surgery following protein immunoblotting analysis [14]. However, to the best of our knowledge, no studies have investigated the sequential activation of the AMPA receptors and glial cells following disc herniation. Therefore, we aimed to investigate the time-dependent changes associated with the activation of the AMPA receptors and glial cells in the spinal dorsal horn after the implantation of NP in the rat model of disc herniation.

\section{MATERIALS AND METHODS}

\section{Animals}

Eighty-three Sprague-Dawley rats (200-250 g) were randomly assigned to the control, sham-operation, and NPexposed groups (Fig. 1). For the analysis of AMPA receptor activity, the rats $(n=40)$ were assigned to the control $(n=6)$, sham-operation $(n=4)$, and NP-exposed $(n=30)$ groups. For assessing the immunohistochemical expression of OX-42 and glial fibrillary acidic protein (GFAP), the rats $(n=40)$ were assigned to the control $(n=10)$ and NP-exposed $(n=30)$ groups. Moreover, three rats were used to investigate the progression of neuropathic pain. They were housed, two per cage, with diurnal light-dark cycles, and food pellets and water were provided ad libitum.

\section{Lumbar disc herniation operation}

All experimental procedures were conducted according to the guidelines of the Institutional Animal Care and Use Committee at Yeungnam University, Republic of Korea (No. YUMC-2017-010). The rats were anesthetized through the intraperitoneal injection of Zoletil $50 \mathrm{mg} /$ kg (Virbac, Carros, France). With the animals placed in the prone position, a midline dorsal incision was made within the pelvic area, and the multifidus muscles were separated along the L4-S1 spinous processes. Laminectomy was performed to expose the left L5 nerve roots and DRG. An incision, approximately $1 \mathrm{~cm}$ in length, between 

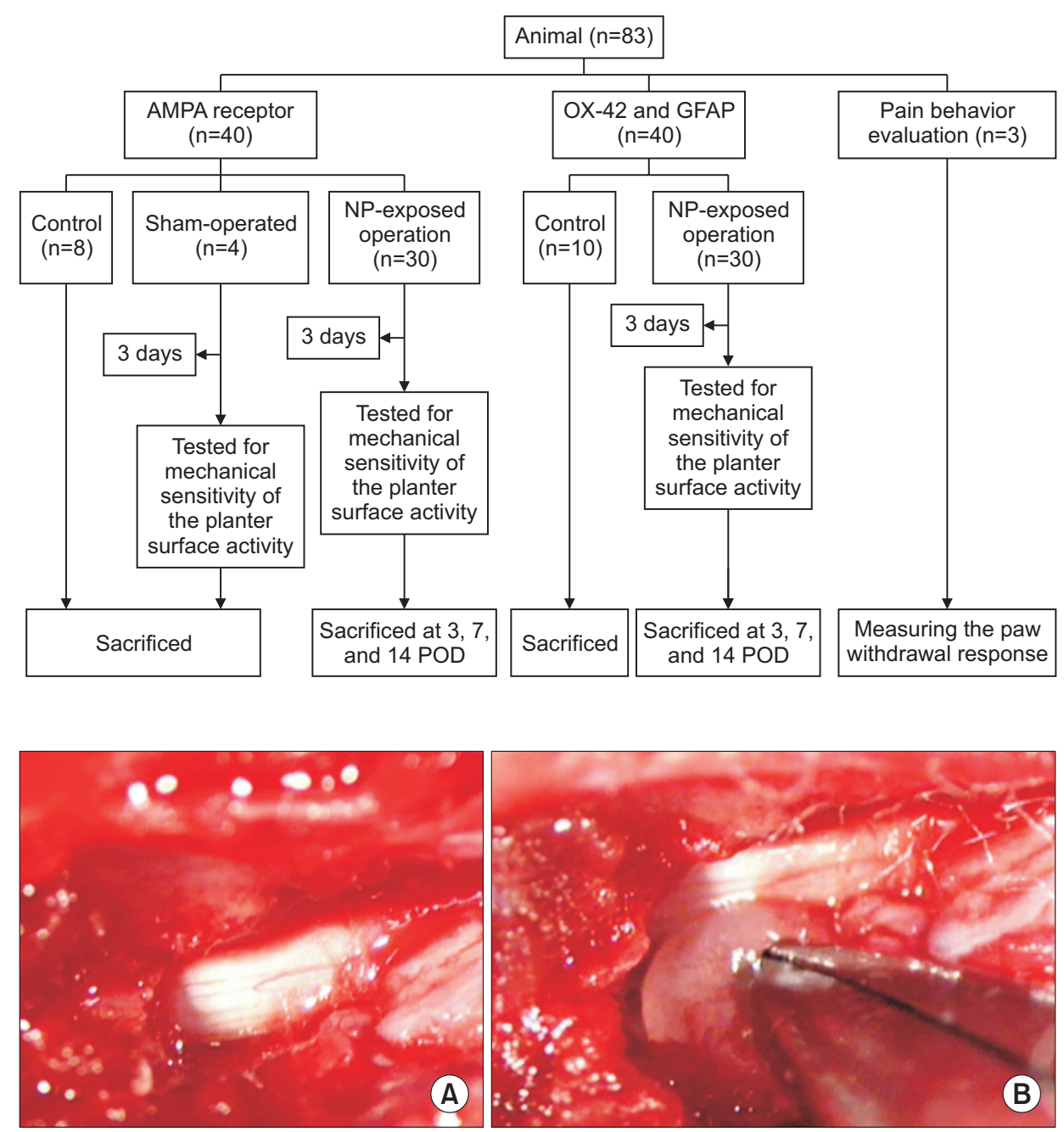

Fig. 1. Study flow schematic for immunohistochemical examination. AMPA, alpha-amino-3-hydroxy-5-methyl-4-isoxazolepropionic acid; GFAP, glial fibrillary acidic protein; NP, nucleus pulposus; POD, postoperative day.

Fig. 2. In the nucleus pulposusexposed group, the left L5 nerve roots were exposed after hemilaminectomy and facetectomy (A) and the nucleus pulposus harvested from coccygeal disc was implanted in the exposed nerve roots without mechanical compression (B). the second and third coccygeal vertebrae of each tail was made on the dorsal surface of the proximal tail for harvesting the autologous NP (Fig. 2). The NP was harvested, using a curette, and implanted beside the left L5 nerve root, immediately proximal to the DRG. The sham surgery was performed similarly, but without the implantation of autologous NP in the left L5 nerve root.

\section{Pain behavior evaluation}

Mechanical allodynia was determined by measuring the paw withdrawal response to the mechanical stimulation of the ipsilateral hind paws with von Frey filaments (North Coast Medical Inc., Morgan Hill, CA, USA); it was calibrated in grams. The rats were placed in a clear plastic cage with a metal mesh floor and permitted to adapt to the testing environment for 15 minutes, and the plantar surface of each hind paw was stimulated to cause a slight bending of the von Frey filaments for 3-5 seconds. The filaments were stimulated in the order of increasing and decreasing thicknesses, beginning with a $0.1 \mathrm{~g}$ probe, and the stimulation was maintained until a consistent withdrawal response to $>3$ out of 5 stimuli was observed. If no withdrawal response was elicited by the $15 \mathrm{~g}$ filament, the mechanical threshold was set at $15 \mathrm{~g}$. The mechanical allodynia at the plantar surfaces of the ipsilateral hind paws was assessed three times a week until the animals were sacrificed. Three rats were tested for up to 38 days postoperatively to identify the pattern of progression of neuropathic pain (Fig. 3).

\section{Immunohistochemical examination}

Five rats each were euthanized 3, 7, and 14 days after surgery for the immunohistochemical staining of the spinal cord for the GluA1 and GluA2 subunits of the AMPA receptor OX-42 and GFAP. The rats in the control group were euthanized without performing sham surgery. 


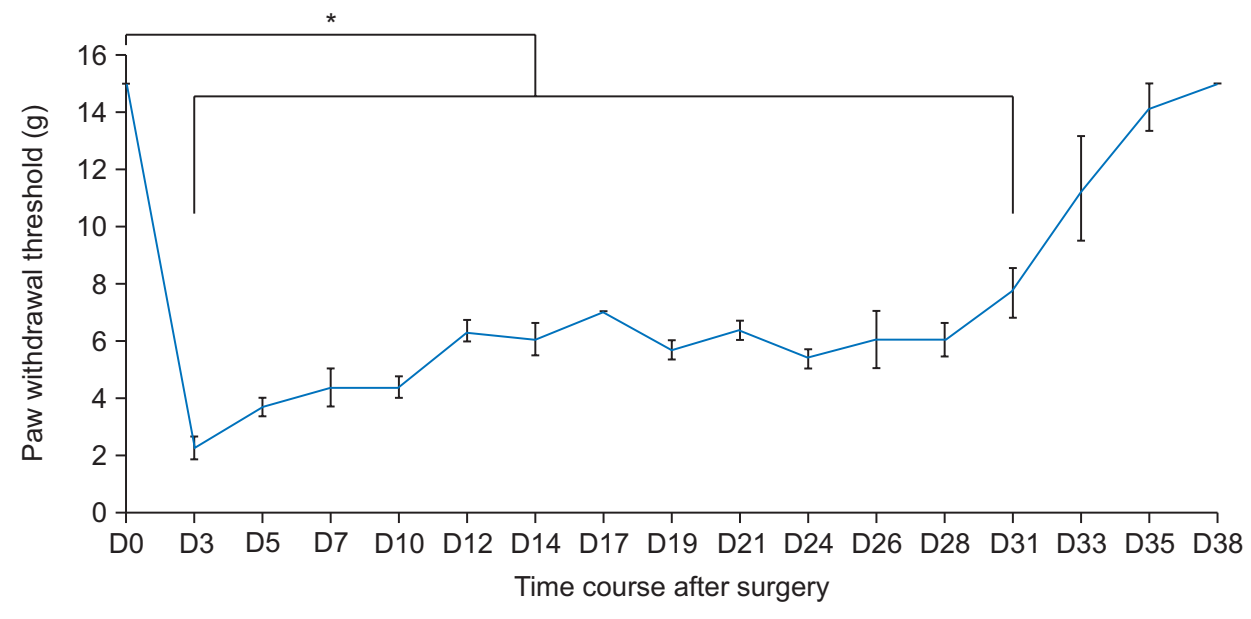

Fig. 3. Time course of neuropathic pain behaviors. Hyperalgesia in a rat model of lumbar disc herniation induced by implantation of the nucleus pulposus to the left L5 nerve root. The withdrawal threshold in the ipsilateral paw is noted at 3 to 38 days following surgery. D, postoperative day. ${ }^{*} \mathrm{p}<0.05$ versus the baseline.
Under anesthesia, a catheter was inserted from the left ventricle of the heart to the aorta, and the animals were perfused with heparinized saline followed by perfusion with $500 \mathrm{~mL}$ of $4 \%$ paraformaldehyde in phosphate buffer (0.1 M, pH 7.4). The L5 spinal cord segment was excised and fixed for 24 hours with the perfused fixative, and the tissue samples were cryoprotected in $30 \%$ sucrose made in phosphate buffer. Transverse sections of the spinal cord (30- $\mu \mathrm{m}$ thick) were sliced on a cryostat and blocked with $10 \%$ normal donkey serum (Jackson ImmunoResearch Laboratories Inc., West Grove, PA, USA) in $0.01 \mathrm{M}$ phosphate-buffered saline (PBS) containing $1 \%$ Triton $\mathrm{X}-100$ for 40 minutes to decrease the non-specific binding of the primary antibody. For performing immunofluorescence microscopy, the sections were incubated overnight at room temperature with a mixture of AntiGluR1 (ABN241, dilution 1:1000; Millipore, Burlington, MA, USA) and anti-GluR2 (AB1768-I, dilution 1:2000; Millipore) antibodies, or with mouse anti-OX-42 (dilution 1:200; Chemicon, Temecula, CA, USA) and anti-GFAP (dilution 1:1000; Dako, Santa Clara, CA, USA) antibodies. After primary antibody incubation, the sections were rinsed with PBS 3 times (5 min/rinse) and incubated with $2 \%$ normal donkey serum for 15 minutes followed by an appropriate combination of Alexa 488-conjugated donkey anti-rabbit secondary antibodies (dilution 1:200; Invitrogen, Eugene, OR, USA) containing 1:200 dilution of Triton X-100 for 2 hours. After several rinses postincubation, the sections were mounted on coverslips covered with a VECTASHIELD Mounting Medium (Vector Laboratories, Burlingame, CA, USA) and observed under a fluorescence microscope (Digital Module R, Leica Microsystems, Switzerland). Images were visualized and captured by a charge-coupled device camera (Olympus, Tokyo, Japan) attached to the microscope.

\section{Quantitative image analysis}

To analyze the immunopositivity of AMPA receptors and glial cells in the spinal dorsal horn, images obtained from the L5 spinal cord sections were captured. One image $(898 \times 660 \mu \mathrm{m})$ captured by the camera was encoded to blind the investigator before analysis. The number of pixels positive for AMPA receptors and glial cells was segmented by applying an appropriate threshold. The intensity of green fluorescence for each frame was obtained using an image analysis software (Leica application suite V4.2; Leica Microsystems, Switzerland). The superficial dorsal horn of the spinal cord had a connection with nociceptive neurons [15]. AMPA receptors are more in the superficial dorsal horn of the spinal cord [16]. AMPA positive and glial cell activity in the superficial dorsal horn were evaluated $[17,18]$. The mean values of each group and the time point versus control mean value in the dorsal horn of the L5 spinal level were calculated as percentages. The numbers of OX-42-positive and GFAP-positive cells were counted using the image analysis software.

\section{Statistical analysis}

Data from each rat were pooled and processed using Excel version 16.26 (Microsoft Corporation, Redmond, WA, USA). The collected data were uploaded into SPSS software (IBM Corporation, Armonk, NY, USA) and analyzed. For comparison, the control values were considered as 100 . Comparisons of the findings from the quantitative image analysis of immunoreactivities and the measurements of mechanical withdrawal thresholds 
were performed using the Kruskal-Wallis test. Statistical significance was set at $\mathrm{p}<0.05$.

\section{RESULTS}

\section{Pain behavior}

The mechanical withdrawal threshold of the control and sham-operated groups was $15 \mathrm{~g}$. Rats that underwent sham surgery did not exhibit a decreased mechanical withdrawal threshold 3 days after surgery. Rats in the NPexposed group that underwent lumbar disc herniation surgery showed statistically significant reductions in the mechanical withdrawal thresholds of the ipsilateral hind paw 3 days after surgery, and these were maintained until postoperative day 31 (Fig. 2).

\section{GluA1 and GluA2 expression}

The immunoreactivities of GluA1 and GluA2 were wellvisualized in the spinal dorsal horn, and they showed intense staining in the superficial lamina of the dorsal horn, the region that received nociceptive input from the periphery (Fig. 4). Compared with the control group, the immunoreactivity of GluAl significantly increased in the animals that underwent surgery within postoperative days 3 to 14 . Three and 7 days after the surgery, the increase in immunoreactivity of GluAl in the spinal dorsal horn was statistically significant (Fig. 5). The immunoreactivity of GluA2 also increased within postoperative days 3 to 7; however, the magnitude of increase was relatively small compared with the immunoreactivity of GluAl. The differences between the immunoreactivities of GluAl and GluA2 at each time point were not statistically significant. Fourteen days after surgery, the increased immunoreac-
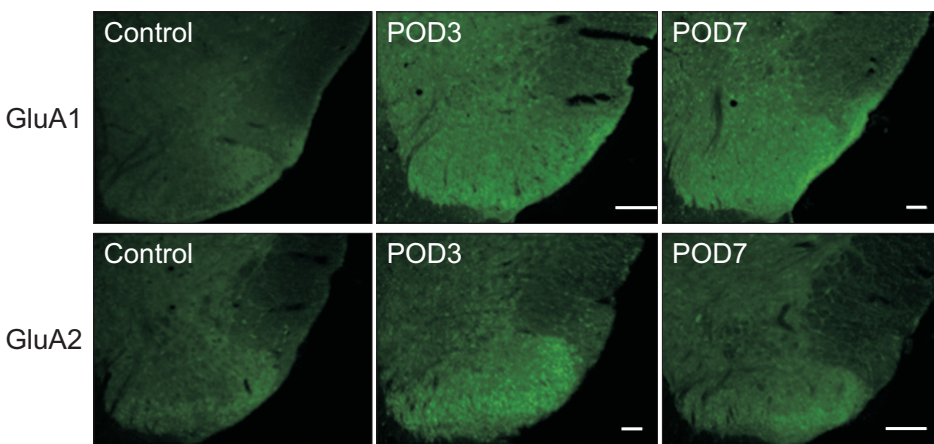

GluA2
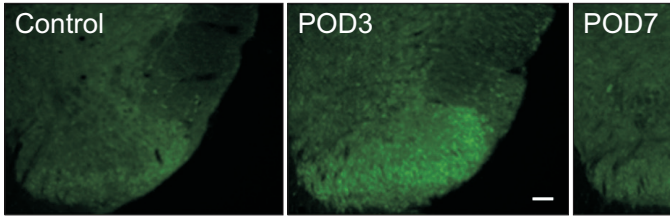

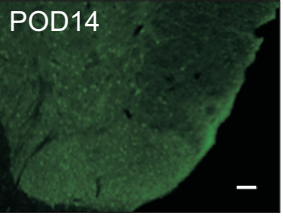

POD14

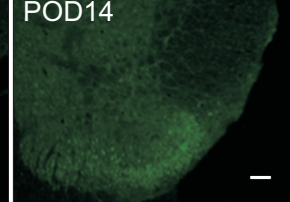

Fig. 4. Immunohistochemical ex pression of GluA1 and GluA2 in the dorsal horn of the L5-level spinal cord before (control), 3, 7, and 14 days after surgery. GluAl are GluA2 are intensely stained in the superficial lamina of the dorsal horn. POD, postoperative day.
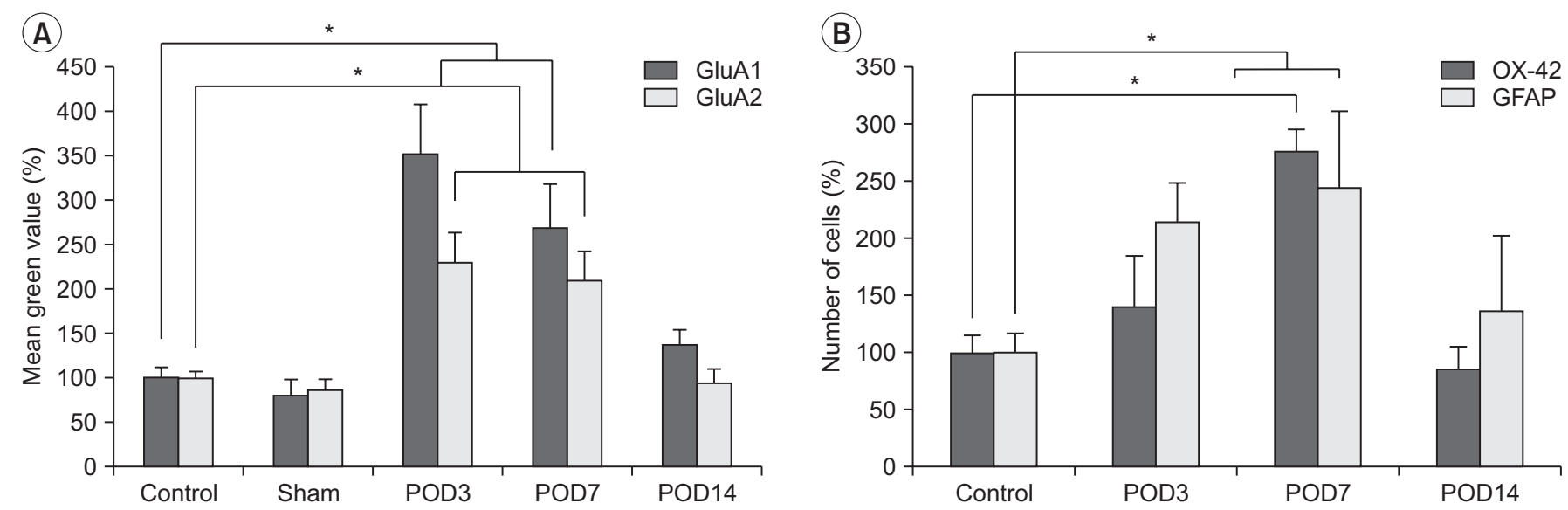

Fig. 5. Semi-quantitative analysis of immunoreactivity of AMPA receptors and microglial cells in the dorsal horn of the spinal cord at postoperative day (POD) 3, 7, and 14. (A) Mean green value of GluA1 and GluA2 is significantly higher at POD3 and POD7, compared with control. (B) Immunoreactivity of OX-42 and GFAP are also increased and return to the control value at 14 days after surgery, with a pattern similar to that of AMPA receptors. For quantification, values and numbers were counted and averaged. Values are presented as mean \pm standard error. AMPA, alpha-amino-3-hydroxy-5-methyl-4-isoxazolepropionic acid; GFAP, glial fibrillary acidic protein. ${ }^{*} \mathrm{p}<0.05$ versus the control group. 

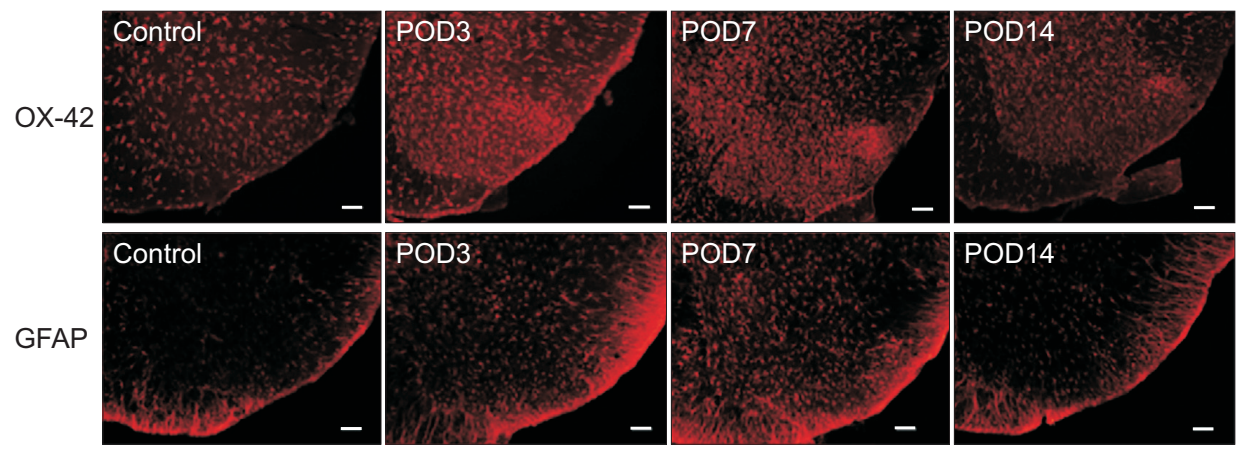

Fig. 6. Immunohistochemical expression of OX-42 and glial fibrillary acidic protein (GFAP) in the dorsal horn of the L5-level spinal cord before (control), 3, 7, and 14 days after surgery. Increased OX-42-positive microglia are noted in superficial ipsilateral dorsal horns at 3 and 7 days following surgery compared with control. GFAP-positive astrocytes are also diffusely elevated in the spinal dorsal horn following surgery. POD, postoperative day.

tivities of the GluAl and GluA2 subunits observed in the rats that underwent surgery gradually normalized to levels observed in homeostatic conditions similar to those of the control group.

\section{OX-42 and GFAP}

Immunohistochemical examination of the spinal dorsal horn for OX-42 and GFAP demonstrated reactive activation of the microglia and astrocytes after surgery (Fig. 6). The immunoreactivity of OX-42 within the superficial lamina of the spinal dorsal horn had significantly increased on postoperative day 7 . The immunoreactivity of GFAP also significantly increased on postoperative days 3 and 7 (Fig. 5). On postoperative day 14, the numbers of OX-42-positive and GFAP-positive cells decreased to levels observed in the control group.

\section{DISCUSSION}

NP is immunogenic; a herniated NP may release substances that induce an autoimmune response and generate chronic inflammation. Inflammation stimulates the nociceptive nerves, which causes nociceptive pain following disc herniation. Moreover, the inflammation of an injured DRG or nerve root promotes the initiation and maintenance of neuropathic pain. Based on this concept, an epidural steroid injection following disc herniation has been accepted as a logical and effective therapeutic option [19]. However, the role and involvement of inflammation throughout the entire process of pain-from the beginning of disc herniation to the chronic neuropathic pain following disc herniation-have not been fully elu- cidated. Establishing a treatment method for radicular pain will facilitate a better understanding of the role of inflammation in nociception, from early nociceptive to and chronic neuropathic pain. Therefore, we investigated the activity of AMPA receptors reciprocally activated by peripheral inflammation and glial cells involved in maintaining chronic neuropathic pain.

Glutamate is an essential excitatory neurotransmitter in the central nervous system. Functional AMPA receptors have been observed in various brain regions and the spinal cord. AMPA receptors are ionotropic glutamate receptors in the post-synaptic membrane that dynamically respond to nociceptive input from peripheral inflammation and contribute to the maintenance of long-lasting transmission of somatosensory input [20,21]. AMPA receptor phosphorylation and upregulation of AMPA increase permeability to $\mathrm{Na}^{+}$and $\mathrm{Ca}^{2+}$, which increases EPSCs $[4,22]$. The upregulation of AMPA receptors is caused by the translocation of AMPA receptors from the cytosol to the plasma membrane. Long-term potentiation (LTP) describes the long-lasting strengthening of synapses based on recent patterns of activity. The AMPA receptor is the main participant in the formation of LTP.

When it comes to the pain, LTP refers to changes in nociception such as hyperalgesia. The continuous increase in EPSC, which is a mechanism of LTP, stimulates central sensitization as in chronic neuropathic pain [4]. AMPA receptors are expressed by neurons and glial cells [23,24]. Classically, astrocytes are characterized by high glutamate transporter activity [25]. Considering that glial cells play an essential role in chronic pain, AMPA receptors play a role in the development of central sensitization of 
the neurons and glial cells within the dorsal horn of the spinal cord.

Although many cells and complex processes result in central sensitization following an insult, we have focused on the AMPA receptor as a surrogate of peripheral inflammation to investigate the duration of inflammation in the process of pain and central sensitization. The rat pain model of lumbar disc herniation showed an increased total level of the AMPA receptor within the dorsal horn. This is a remarkable finding, given those observed for other chronic pain models. Autoimmunity and hypoxia caused by the herniated NP result in inflammation around the herniated disc. Hence, the investigation of the sequential changes in the AMPA receptor levels can facilitate a better understanding of the temporal correlation between the increased EPSCs caused by inflammation and disc herniation.

Contrary to other pain models, a significant increase in the GluA1 and GluA2 subunits of AMPA receptors was observed in the rat pain model of lumbar disc herniation in this study. These increases in immunoreactivity persisted for 3 to 7 days after surgery and returned to baseline 14 days after surgery. The paw withdrawal threshold was reduced after surgery and maintained for 31 days after surgery. AMPA involvement is the highest at the early phase of neuropathic pain, and its role gradually diminishes in chronic neuropathic pain. The AMPA receptor mainly mediates fast EPSCs in dorsal horn neurons. Therefore, peripheral inflammation promotes the upregulation of AMPA receptors, which strengthens the synapses among afferent and second-order nociceptive nerves, interneurons within the dorsal horn, and glial cells. Despite the normalization of GluA1 and GluA2 14 days after surgery, which indicates a decrease in nociceptive input from inflammation, central sensitization facilitated the maintenance of mechanical hypersensitivity after surgery.

Continuous nociceptive input followed by a rise in the AMPA receptor activities increases the action potential of post-synaptic neurons, which promotes central sensitization. Subsequently, central sensitization yields chronic pain, even if there is no further increase in the activity of the AMPA receptors. This activity gradually reduces. Therefore, AMPA receptors play an important role during the period of increased EPSC due to inflammation following disc herniation.

Chen et al. [26] reported that GluA2 protein levels were significantly low in the plasma membrane in the rat model of nerve injury. Moreover, cytosolic GluR2 levels were elevated, and the amount of membrane-bound GluA2 was significantly reduced after 24 hours postFreund's complete adjuvant (CFA) injection [27]. GluA2 is a $\mathrm{Ca}^{2+}$-impermeable ion channel. GluA2-lacking AMPA receptors in the dorsal horn induce an inward rectification of EPSCs, thereby maintaining chronic neuropathic pain. N-methyl-D-aspartate receptors (NMDARs) are critically involved in the induction and maintenance of persistent pain after a noxious event [28]. The activated NMDAR/triggered protein kinase C signaling cascade induces GluR2 internalization in dorsal horn neurons after persistent inflammation [3]. Therefore, the reduction in GluA2 subunits in the plasma membrane after nerve injury or CFA injection constitutes an important mechanism of pain. However, in this study, the immunoreactivity of GluA2 was elevated, likewise the immunoreactivity of GluA1. Although not statistically significant, the magnitude of increase in the immunoreactivity of GluAl was greater than that of GluA2 in this study; therefore, the GluA1/GluA2 ratio was elevated, as reported by a previous study [17].

The hind paw is injected with inflammatory agents to create rat models of inflammatory hyperalgesia. The GluR1 and GluR2 trafficking patterns reportedly vary based on the inflammatory agents injected [29]. Most studies reported no definite change in the total amount within the dorsal horn, except their increased insertions into the membrane. However, these studies have investigated changes in the distribution of GluAl within several minutes or up to 1 day after an insult. Our study revealed the upregulation of GluA1 and GluA2 after 7 days after surgery. Therefore, we speculate that this difference is due to the extended period of inflammation and the location of the inflammation.

Excitatory neurotransmitters released by presynaptic neurons within the dorsal horn activate glial cells. The activation of the Toll-like receptor 4 (TLR4) and P2X4 receptors has been implicated in chronic pain states following peripheral nerve injury [5]. Activated glial cells closely communicate with neurons. Tumor necrosis factor- $\alpha$ (TNF- $\alpha$ ), interleukin- $1 \beta$, and brain-derived neurotrophic factor are released from activated glial cells, and this initiates hyperactivity in dorsal horn cells [6]. Conversely, cellular debris from apoptotic and necrotic 
cells promote phagocytosis of glial cells, resulting in a decrease in pro-inflammatory cytokines [30]. Therefore, activated glial cells play a role in promoting neuropathic pain and have a protective mechanism at the same time.

We found an increase in astrocytes and microglia in the dorsal horn 3 and 7 days following surgery, which returned to control levels on day 14 . Therefore, the activity of glial cells also increased in response to inflammation induced by the implanted NP followed by a gradual decrease. Excessive AMPA receptor activation in astrocytes causes the downregulation of astrocyte GLAST (glutamate aspartate transporter) [31]. GLAST removes glutamate from the extracellular space. The downregulation of astrocyte GLAST aggravates excitotoxic conditions by increasing extracellular glutamate levels. The increased AMPA receptor activation of microglia releases inflammatory mediators and TNF- $\alpha$ and reduces protective mediators [32]. TNF- $\alpha$ produced by microglia stimulates the upregulation of transmembrane AMPA receptors on neurons, which increases their reaction to extracellular glutamate. Therefore, the upregulation of AMPA receptors on glial cells leads to an increase in extracellular glutamate, which has a role in initiating central sensitization.

Our study has several limitations. First, the activation of AMPA receptors is not a direct and precise surrogate of peripheral inflammation following disc herniation. However, the degrees of activity of various cytokines and immune cells during inflammation are modified depending on the time. Moreover, we aimed to investigate the changes in the central, and not the peripheral, nervous system. Hence, we selected AMPA receptors to indicate the activity of the central nervous system in response to peripheral inflammation following the surgery. Second, we conducted immunohistochemistry only for measuring AMPA receptor levels. Hence the mechanisms underlying an increase in AMPA receptors and those underlying its increase in production or decrease in degradation need to be elucidated.

In summary, in the rat pain model of lumbar disc herniation, increased immunoreactivity of AMPA receptors (GluA1 and GluA2 subunits) and glial cells (microglia and astrocyte) was observed on days 3 and 7 after surgery, and they decreased to control levels after 14 days. Therefore, AMPA receptor activation and gliosis caused by inflammation after disc herniation play roles in the in- duction of central sensitization.

\section{CONFLICT OF INTEREST}

No potential conflict of interest relevant to this article was reported.

\section{ACKNOWLEDGMENTS}

This work was supported by the National Research Foundation of Korea (NRF) grant funded by the Korean government (MSIT) (No. NRF-2017R1C1B5018094).

\section{AUTHOR CONTRIBUTION}

Conceptualization: Lee DG, Kwak DG. Methodology: Lee DG. Formal analysis: Lee DG, Kwak DG. Project administration: Lee DG, Kwak DG. Visualization: Kwak DG. Writing - original draft: Kwak DG. Writing - review and editing: Lee DG. Approval of final manuscript: all authors.

\section{REFERENCES}

1. Garry EM, Fleetwood-Walker SM. A new view on how AMPA receptors and their interacting proteins mediate neuropathic pain. Pain 2004;109:210-3.

2. Kopach O, Voitenko N. Extrasynaptic AMPA receptors in the dorsal horn: evidence and functional significance. Brain Res Bull 2013;93:47-56.

3. Park JS, Voitenko N, Petralia RS, Guan X, Xu JT, Steinberg JP, et al. Persistent inflammation induces GluR2 internalization via NMDA receptor-triggered PKC activation in dorsal horn neurons. J Neurosci 2009;29:3206-19.

4. Kopach O, Kao SC, Petralia RS, Belan P, Tao YX, Voitenko N. Inflammation alters trafficking of extrasynaptic AMPA receptors in tonically firing lamina II neurons of the rat spinal dorsal horn. Pain 2011;152:912-23.

5. Tsuda M. Microglia in the spinal cord and neuropathic pain. J Diabetes Investig 2016;7:17-26.

6. Taves S, Berta T, Chen G, Ji RR. Microglia and spinal cord synaptic plasticity in persistent pain. Neural Plast 2013;2013:753656.

7. Milligan ED, Watkins LR. Pathological and protec- 
tive roles of glia in chronic pain. Nat Rev Neurosci 2009;10:23-36.

8. Mulleman D, Mammou S, Griffoul I, Watier H, Goupille P. Pathophysiology of disk-related sciatica. I.--Evidence supporting a chemical component. Joint Bone Spine 2006;73:151-8.

9. Sasaki N, Sekiguchi M, Shishido H, Kikuchi S, Yabuki S, Konno S. A comparison of pain-related behavior following local application of nucleus pulposus and/or mechanical compression on the dorsal root ganglion. Fukushima J Med Sci 2011;57:46-53.

10. Tachihara H, Sekiguchi M, Kikuchi S, Konno S. Do corticosteroids produce additional benefit in nerve root infiltration for lumbar disc herniation? Spine (Phila Pa 1976) 2008;33:743-7.

11. Uesugi K, Sekiguchi M, Kikuchi S, Konno S. The effect of repeated restraint stress in pain-related behavior induced by nucleus pulposus applied on the nerve root in rats. Eur Spine J 2011;20:1885-91.

12. Park HW, Ahn SH, Son JY, Kim SJ, Hwang SJ, Cho YW, et al. Pulsed radiofrequency application reduced mechanical hypersensitivity and microglial expression in neuropathic pain model. Pain Med 2012;13:1227-34.

13. Gold MS, Gebhart GF. Nociceptor sensitization in pain pathogenesis. Nat Med 2010;16:1248-57.

14. Cho JH, Lee DG. Translocation of AMPA receptors in the dorsal horn of the spinal cord corresponding to long-term depression following pulsed radiofrequency stimulation at the dorsal root ganglion. Pain Med 2019:pnz307.

15. Basbaum AI, Bautista DM, Scherrer G, Julius D. Cellular and molecular mechanisms of pain. Cell 2009;139:267-84.

16. Engelman HS, Allen TB, MacDermott AB. The distribution of neurons expressing calcium-permeable AMPA receptors in the superficial laminae of the spinal cord dorsal horn. J Neurosci 1999;19:2081-9.

17. Wigerblad G, Huie JR, Yin HZ, Leinders M, Pritchard RA, Koehrn FJ, et al. Inflammation-induced GluA1 trafficking and membrane insertion of $\mathrm{Ca} 2+$ permeable AMPA receptors in dorsal horn neurons is dependent on spinal tumor necrosis factor, PI3 kinase and protein kinase A. Exp Neurol 2017;293:144-58.

18. Kim KJ, Lee SH, Hwang SJ, Oh SJ. Expression of pERK in the neurons and microglia in the spinal cord of the rat with neuropathic pain. J Korean Neurotraumatol
Soc 2005;1:19-29.

19. Buenaventura RM, Datta S, Abdi S, Smith HS. Systematic review of therapeutic lumbar transforaminal epidural steroid injections. Pain Physician 2009;12:23351.

20. Gong K, Kung LH, Magni G, Bhargava A, Jasmin L. Increased response to glutamate in small diameter dorsal root ganglion neurons after sciatic nerve injury. PLoS One 2014;9:e95491.

21. Youn DH, Gerber G, Sather WA. Ionotropic glutamate receptors and voltage-gated $\mathrm{Ca} 2+$ channels in longterm potentiation of spinal dorsal horn synapses and pain hypersensitivity. Neural Plast 2013;2013:654257.

22. Leem JW, Kim HK, Hulsebosch CE, Gwak YS. Ionotropic glutamate receptors contribute to maintained neuronal hyperexcitability following spinal cord injury in rats. Exp Neurol 2010;224:321-4.

23. Verkhratsky A, Kirchhoff F. Glutamate-mediated neuronal-glial transmission. J Anat 2007;210:651-60.

24. Wang Y, Wu J, Wu Z, Lin Q, Yue Y, Fang L. Regulation of AMPA receptors in spinal nociception. Mol Pain 2010;6:5.

25. Matthias K, Kirchhoff F, Seifert G, Huttmann K, Matyash $\mathrm{M}$, Kettenmann $\mathrm{H}$, et al. Segregated expression of AMPA-type glutamate receptors and glutamate transporters defines distinct astrocyte populations in the mouse hippocampus. J Neurosci 2003;23:1750-8.

26. Chen SR, Zhou HY, Byun HS, Pan HL. Nerve injury increases GluA2-lacking AMPA receptor prevalence in spinal cords: functional significance and signaling mechanisms. J Pharmacol Exp Ther 2013;347:765-72.

27. Park JS, Yaster M, Guan X, Xu JT, Shih MH, Guan Y, et al. Role of spinal cord alpha-amino-3-hydroxy5-methyl-4-isoxazolepropionic acid receptors in complete Freund's adjuvant-induced inflammatory pain. Mol Pain 2008;4:67.

28. Petrenko AB, Yamakura T, Baba H, Shimoji K. The role of N-methyl-D-aspartate (NMDA) receptors in pain: a review. Anesth Analg 2003;97:1108-16.

29. Tao YX. Dorsal horn alpha-amino-3-hydroxy-5-methyl-4-isoxazolepropionic acid receptor trafficking in inflammatory pain. Anesthesiology 2010;112:1259-65.

30. Elward K, Gasque P. "Eat me" and "don't eat me" signals govern the innate immune response and tissue repair in the CNS: emphasis on the critical role of the complement system. Mol Immunol 2003;40:85-94. 
31. Lopez-Bayghen E, Espinoza-Rojo M, Ortega A. Glutamate down-regulates GLAST expression through AMPA receptors in Bergmann glial cells. Brain Res Mol Brain Res 2003;115:1-9.
32. Sivakumar V, Ling EA, Lu J, Kaur C. Role of glutamate and its receptors and insulin-like growth factors in hypoxia induced periventricular white matter injury. Glia 2010;58:507-23. 